\title{
Deep winds on Jupiter as measured by the Galileo probe
}

\section{David H. Atkinson $*$, Andrew P. Ingersoll $\dagger$ \& Alvin Seiff}

${ }^{*}$ Department of Electrical Engineering, University of Idaho, Moscow, Idaho 83844, USA

$\dagger$ Division of Geological and Planetary Sciences, California Institute of Technology, Pasadena, California 91125, USA

$¥$ San Jose State University Foundation, NASA Ames Research Center, Moffett Field, California 94035, USA

The Doppler Wind Experiment on the Galileo probe provided the first in situ data on wind speeds in Jupiter's atmosphere. Initial analysis $^{1}$ of the results indicated that wind speeds increase with depth, rather than decaying to zero below the cloud tops or remaining relatively constant as had previously been assumed ${ }^{2}$. But this earlier analysis was subject to several potential sources of error, as highlighted by the fact that wind speeds measured at the cloud tops did not seem to match those inferred from tracking clouds $s^{3}$ in images obtained by the Voyager spacecraft. Here we report new analyses of the probe data that use a corrected treatment of the timing errors, adopt the measured ${ }^{4}$ (rather than predicted) descent trajectory, and incorporate a new calibration of the instrumentation that takes into account the unexpectedly high temperatures encountered by the probe. We determine wind speeds at the cloud tops (700-mbar level) in the range 80$100 \mathrm{~m} \mathrm{~s}^{-1}$, in agreement with the results of cloud tracking; the speed increases dramatically between 1 and 4 bar, and then remains nearly constant at $\sim 170 \mathrm{~m} \mathrm{~s}^{-1}$ down to the 21 -bar level. The increase in wind speed implies a latitudinal density gradient of $0.5 \%$ per degree in the 1-2 bar altitude range, but whether these winds are driven by internal heat or absorbed sunlight remains uncertain.

On arrival at Jupiter on 7 December 1995, the Galileo probe telemetered 57.5 minutes of atmospheric measurements to the orbiter. The probe telemetry frequency, measured on the orbiter, was altered from the transmitted frequency primarily by Doppler contributions from the orbiter trajectory, the probe trajectory (from descent and from the rotation of Jupiter) and the winds. When the nominal trajectories are removed from the measured frequency profile, the Doppler frequency residuals are attributed primarily to the effect of local zonal winds. To convert the Doppler residuals into a wind profile, an assumption must be made about the wind direction. At the latitude of probe entry, near $6.5^{\circ}$ north, the meridional winds are an order of magnitude smaller than the zonal winds ${ }^{5}$ and, by considerations of continuity, the vertical winds are expected to be three orders of magnitude smaller. It is therefore reasonable to project the frequency residuals onto the probe local east-west direction. Inversion of the frequency residuals, including accounting for the change in probe longitude $\left(\sim 0.45^{\circ}\right)$ due to the integrated wind effect, yields a profile of zonal winds at the probe descent location. This profile, when combined with the nominal trajectory, provides a least-squares best fit to the measured frequency profile.

The early results from the Doppler Wind Experiment (DWE) presented several problems. First, the wind speed at the top of the clouds did not match the speed reported from tracking clouds in Voyager images ${ }^{3}$ or from tracking the large-scale features at the time of probe entry ${ }^{6}$. The DWE gave a wind speed of $175 \pm 25 \mathrm{~m} \mathrm{~s}^{-1}$ at cloud-top altitude. Tracking of the clouds at the latitude of the probe generally gives a value of $100-105 \mathrm{~m} \mathrm{~s}^{-1}$, although tracking small features sometimes gives speeds up to $160 \mathrm{~ms}^{-1}$ (ref. 7). Second, by the end of the descent mission the probe interior reached a temperature of $150^{\circ} \mathrm{C}$, significantly higher than the maximum expected operating temperature of $60^{\circ} \mathrm{C}$. The high temperatures might have caused unmodelled frequency shifts of the ultrastable oscillator leading to systematic errors in the derived velocities. Third, timing errors between the relay receiver hardware and the orbiter were modelled incorrectly. Last, the early DWE analysis used the predicted descent velocity of the probe rather than the measured descent velocity because the latter was not available owing to the higher-than-expected temperatures. As the descent velocity causes a major Doppler shift that must be removed before the Doppler shift due to the zonal winds can be measured, this was another potential source of error.

Based on the measured Doppler frequencies and improved analysis as described above, the retrieved zonal winds in the upper atmosphere are now in close agreement with cloud-top imaging from Voyager 2 (ref. 3). Near the 700-mbar level, the Dopplermeasured winds are $90 \mathrm{~m} \mathrm{~s}^{-1}$. Between 1 bar and 4 bar the zonal wind speed increases rapidly, an effect also seen in the wind determinations by the accelerometers ${ }^{8}$ of the Atmospheric Structure Instrument and the Earth-based Doppler measurements ${ }^{9}$. From 4 bar to 21 bar, the winds remain relatively constant near $170 \mathrm{~m} \mathrm{~s}^{-1}$ (Fig. 1).

An error envelope spanning a range of possible wind profiles is shown in Fig. 1; it includes descent velocities that vary from $95 \%$ to $105 \%$ of nominal at each time point, probe entry and initial descent longitudes within $\pm 0.21^{\circ}(3 \sigma)$ of nominal, and assuming the

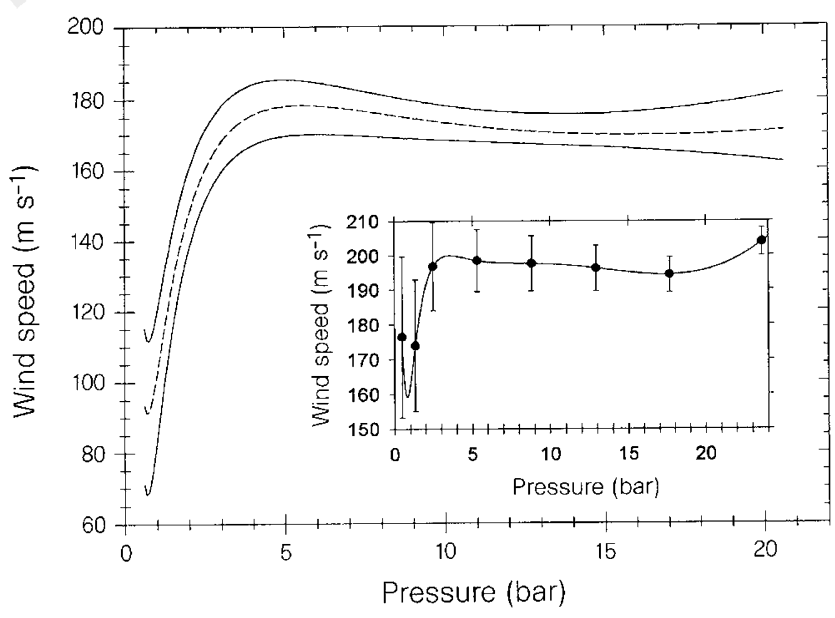

Figure 1 The Doppler wind measurements (main figure) now incorporate a number of significant changes from the preliminary results of Atkinson et al. ${ }^{1}$ (inset). The new wind speeds were calculated using the probe descent velocity profile obtained from measurements of pressure and temperature by the Atmospheric Structure Instrument, the assumption of hydrostatic equilibrium, and the equation of state. Descent velocities are now modelled to better than $5 \%$ $\left(\sim 1-5 \mathrm{~m} \mathrm{~s}^{-1}\right)$ throughout the descent, and the probe entry and initial descent longitude is known to $\pm 0.21^{\circ}(3 \sigma)$. Periodic null frequency measurements are thought to be caused by imperfections in the relative timing of the relay receiver hardware and orbiter clocks. When unaccounted for, the timing mismatch between the relay receiver hardware and the orbiter ultrastable oscillator (USO) causes periodic discontinuities of $\sim 7 \mathrm{~Hz}$ in the measured frequencies. Our treatment of the timing errors involves averaging through, as opposed to complete removal of, the discontinuities, resulting in a net reduction of the frequency residuals by $\sim 50 \mathrm{~Hz}$ and a subsequent decrease in the retrieved deep zonal winds of $30 \mathrm{~m} \mathrm{~s}^{-1}$. The probe USO, qualification-tested to $60^{\circ} \mathrm{C}$ and acceptancetested to $50^{\circ} \mathrm{C}$, in fact experienced temperatures nearing $150^{\circ} \mathrm{C}$ at the end of probe transmission. Thermal tests at NASA Ames Research Center on several flight spare USOs have recently concluded; the high-temperature behaviour of the USOs has been characterized and incorporated into the probe oscillator frequency model. The error envelope (solid lines) of wind speeds is based on worstcase descent velocity variations $( \pm 5 \%)$, probe longitude error $\left( \pm 0.21^{\circ}, 3 \sigma\right)$ and USO thermal response corrections (none/maximum). 
minimum (no thermal corrections) and maximum thermal response models of the probe ultrastable oscillator. Given our current understanding of the high-temperature response of this oscillator, it is now seen that the tendency reported previously ${ }^{1}-$ towards increasing winds in deepest regions explored by the probe-has disappeared.

The above-mentioned wind measurements by the Atmospheric Structure Instrument's accelerometers ${ }^{8}$ are particularly reassuring in that they are obtained by an entirely different technique that does not depend on radio tracking, the stability of a crystal oscillator, or the substraction of other contributing velocities. They are of low velocity resolution and limited accuracy, however, and therefore do not give precise wind speeds. The radio tracking measurements from Earth (by the Very Large Array) have a much better viewing angle than that available from the overhead Galileo orbiter, but were significantly affected by the unknown frequency offset of the probe ultrastable oscillator and the enormous transmission distance leaving only a faint signal at Earth which must be extracted from the noise ${ }^{9}$. It is remarkable that these three techniques agree on the essential description of the winds, leaving little doubt that they have been characterized.

Although there was speculation about whether the winds were shallow or deep, very few had predicted that the winds would increase with depth. By 'shallow' one usually means 'above the base of the water cloud', that is, above the 5-bar level. This is the region where sunlight is absorbed and latent heat is released, so it was assumed that if the winds drew their energy from these sources they should decay with depth below the cloud base $e^{2,10-12}$. On the other hand, if the winds drew their energy from internal heat, they might continue down indefinitely into the fluid interior of Jupiter ${ }^{13-15}$. Only one analysis ${ }^{16}$ predicted that the winds would increase with depth. The DWE has shown that the winds are 'deep'. But it is not clear that this means that they are powered by internal heat; neither is it clear why the winds increase with depth at the 1-4 bar level.

In the atmospheres of rotating planets, the variation of zonal wind with depth is proportional to the variation of density with latitude. The density gradient is taken at constant pressure. If the gradient is zero, the fluid is said to be barotropic. If the interior of a fluid planet is barotropic, then each cylinder, concentric with the axis of rotation, will rotate as a solid body. These statements, proved nearly a century ago ${ }^{17}$, are valid when forcing and dissipation are small. The forcing that maintains the winds may be confined to the surface layers or it may be distributed throughout the fluid. Thus we cannot say with certainty that the zonal winds are powered by internal heat.

The fluid is clearly not barotropic in the 1-4 bar pressure range where zonal wind is increasing with depth. The increase implies a density decrease of $0.5 \%$ per degree of latitude at the $1-2$ bar levels. This is three to four times larger than, but of the same sign as, that inferred from the temperature gradients observed at the 150- and 270 -mbar levels ${ }^{18}$. The latter have been interpreted as a sign of upwelling to the south, and downwelling to the north, of the probe site. The DWE results suggest that the upwellings and downwellings extend at least to the 4-bar level.

The constancy of the wind in the 4-21 bar range implies that the fluid there is barotropic. This is expected, as sunlight does not penetrate ${ }^{19}$ and latent heat is not released at these levels. Convection and radiation, the other modes of heat transfer, are likely to maintain a barotropic state $e^{20}$. Thus the $170 \mathrm{~m} \mathrm{~s}^{-1}$ winds measured by the DWE probably extend well into the fluid interior of Jupiter. This result is qualitatively consistent with studies of cloud-top winds, whose vorticity can be used to infer the winds at depth ${ }^{21}$. Magnetic fields ${ }^{22}$, phase transitions, and small gradients of temperature can alter the barotropic state, so the zonal winds do not necessarily extend throughout the planet. But the DWE result shows that the winds are deep, and this has important implications for atmospheric dynamics.
Received 7 March; accepted 3 June 1997.

1. Atkinson, D. H., Pollack, J. B. \& Seiff, A. Galileo Doppler measurements of the deep zonal winds at Jupiter. Science 272, 842-843 (1996).

2. Pollack, J. B., Atkinson, D. H., Seiff, A. \& Anderson, J. D. Retrieval of a wind profile from the Galileo probe telemetry signal. Space Sci. Rev. 60, 143-178 (1992).

3. Limaye, S. S. Jupiter: new estimates of the mean zonal flow at the cloud level. Icarus 65, 335-352 (1986)

4. Seiff, A. et al. Structure of the atmosphere of Jupiter: Galileo probe measurements. Science 272, 844845 (1996).

Ingersoll, A. P. et al. Interaction of eddies and mean zonal flow on Jupiter as inferred from Voyager 1 and 2 images. J. Geophys. Res. 86, 8733-8743 (1981).

6. Orton, G. et al. Earth-based observations of the Galileo probe entry site. Science 272, 839-840 (1996).

7. Beebe, R. F., Simon, A. A. \& Huber, L. F. Comparison of Galileo probe and Earth-based translation rates of Jupiter's equatorial clouds. Science 272, 841 (1996)

Seiff, A. et al. Wind speeds measured in the deep jovian atmosphere by the Galileo probe accelerometers. Nature 388, 650-652 (1997).

9. Folkner, W. M. et al. Earth-based radio tracking of the Galileo probe for Jupiter wind estimation. Science 275, 644-646 (1997).

10. Hess, S. L. \& Panofsky, H. A. in Compendium of Meteorology (ed. Malone, T. F.) 391-398 (Am. Meteorol. Soc., Boston, 1951)

11. Ingersoll, A. P. \& Cuzzi, J. N. Dynamics of Jupiter's cloud bands. J. Atmos. Sci. 26, 981-985 (1969).

12. Williams, G. P. Jovian dynamics. Part I: vortex stability, structure, and genesis. J. Atmos. Sci. 53, 26852734 (1996).

13. Ingersoll, A. P. \& Cuong, P.-G. Numerical model of long-lived jovian vortices. J. Atmos. Sci. 38, $2067-$ 2076 (1981).

14. Busse, F. H. A simple model of convection in the jovian atmosphere. Icarus 29, 255-260 (1976).

15. Ingersoll, A. P. \& Pollard, D. Motion in the interiors and atmospheres of Jupiter and Saturn: scale analysis, anelastic equations, barotropic stability criterion. J. Atmos. Sci. 52, 62-80 (1982)

16. Dowling, T. E. Estimate of Jupiter's deep zonal-wind profile from Shoemaker-Levy 9 data and Arnol'd's second stability criterion. Icarus 117, 439-442 (1995).

17. Poincaré, H. Sur la Précession des corps déformables. Bull. Astron. 27, 231-356 (1910).

18. Gierasch, P. J., Conrath, B. J. \& Magalhaes, J. O. Zonal mean properties of Jupiter's upper troposphere from Voyager infrared observations. Icarus 67, 456-483 (1986).

19. Sromovsky, L. A. et al. Solar and thermal radiation in Jupiter's atmosphere: initial results of the Galileo probe net flux radiometer. Science 272, 851-854 (1996).

20. Ingersoll, A. P. \& Porco, C. C. Solar heating and internal heat flow on Jupiter. Icarus 35, $27-43$ (1978)

21. Dowling, T. E. \& Ingersoll, A. P. Jupiter's great red spot as a shallow water system. J. Atmos. Sci. 46, 3256-3278 (1989).

22. Kirk, R. L. \& Stevenson, D. J. Hydromagnetic constraints on deep zonal flow in the giant planets. Astrophys. J. 316, 836-846 (1987).

Acknowledgements. We thank the Galileo Project and NASA's Planetary Atmospheres Program. This work was supported by NASA.

Correspondence and requests for materials should be addressed to D.H.A. (e-mail: atkinson@maxwell. ee.uidaho.edu)

\section{Wind speeds measured in the deep jovian atmosphere by the Galileo probe accelerometers}

\section{A. Seiff $¥ \uparrow$, R. C. Blanchard $\ddagger$, T. C. D. Knight $₫$, G. Schubert",} D. B. Kirk $₫$, D. Atkinsong, J. D. Mihalov ${ }^{\star} \&$ R. E. Young*

${ }^{\star}$ NASA-Ames Research Center, Moffett Field, California 94035, USA

$\dagger$ Department of Meteorology, San Jose State University, San Jose, California, USA $\ddagger$ NASA Langley Field, MS-107, Hampton, Virginia 23665, USA

$\|$ Department of Earth \& Space Science, UCLA, Los Angeles, California 90024, USA

Department of Electrical Engineering, University of Idaho, Moscow,

Idaho 83844, USA

The atmosphere of Jupiter has a complex circulation which, until recently, has been observable only at the cloud tops ${ }^{1,2}$; the mechanisms driving the winds, and the nature of the interior circulation, remained unknown ${ }^{3}$. Recent analyses ${ }^{4-6}$ of the radio signal from the Galileo probe, obtained during its descent into the jovian atmosphere, have suggested a vigorous interior circulation below the 4-bar level. Here we report an independent measurement of the winds below the cloud tops, making use of the data obtained by the two accelerometers on the descending probe. We find evidence for two distinct wind regimes, in general agreement with the Doppler radio measurements: a region of wind shear between 1 and 4 bar, where the wind speed increases dramatically with depth; and then a region of constant high-velocity winds down to at least the 17-bar level.

We present here acceleration data and their interpretation to 